\title{
Efecto del colesterol y la dimetilformamida sobre parámetros posdescongelación en espermatozoides de caballos criollos colombianos
}

\author{
Effect of cholesterol and dimethiyl-formamide on post-thawing \\ parameters in Colombian creole stallion sperm
}

Ana M Mesa, ${ }^{1 *}$ M.Sc., Guillermo Henao ${ }^{2}{ }^{2}$ M.Sc.

\begin{abstract}
1Universidad Nacional de Colombia, Sede Medellín, Facultad de Ciencias, Maestría CienciasBiotecnología, Grupo Biogem. 2Universidad Nacional de Colombia Sede Medellín, Facultad de ciencias agropecuarias, Departamento de Producción Animal, Grupo Biotecnología de la Reproducción. *Correspondencia: mesa.anamaria@gmail.com
\end{abstract}

Recibido: Noviembre de 2010; Aceptado: Octubre de 2011.

\section{RESUMEN}

Objetivo. Evaluar el efecto del colesterol y la dimetilformamida (DMF) sobre la criosupervivencia del semen de caballos criollos colombianos. Materiales y métodos. Se recolectó semen de diez caballos y se congeló bajo el mismo protocolo, con variaciones del crioprotector (glicerol 5\% o DMF $5 \%$ ) y la adición o ausencia de colesterol como modificador de membrana ( $1.5 \mathrm{mg}$ ciclodextrinas con colesterol por cada $120 \times 10^{6}$ células). La criosupervivencia se evaluó estimando movilidad mediante el software SCA. La vitalidad e integridad de membrana posdescongelación se estimó usando eosina-nigrosina y test hipo-osmótico respectivamente. Resultados. Incubar el semen con el colesterol, tuvo un aumento significativo del porcentaje de movilidad total y progresiva, en la velocidad de los espermatozoides y el porcentaje de espermatozoides rápidos y una disminución del porcentaje de espermatozoides con movilidad lenta. Adicionalmente se incrementó el porcentaje de espermatozoides vivos y con integridad de membrana $(p<0.05)$. La DMF como agente crioprotector, mejoró todos los parametros evaluados al ser comparada el glicerol $(p<0.05)$. Conclusiones. Ambos procedimientos mejoraron los parámetros evaluados debido a efectos aditivos del crioprotector y del modificador de membrana, pero no hubo interacción entre estos dos factores.

Palabras clave: Colesterol, criopreservación, dimetilformamida (Fuente:CAB).

\begin{abstract}
Objective. To evaluate the effect of cholesterol and dimethyl formamide on the cryosurvival of colombian creole stallion sperm. Materials and methods. Semen from ten stallions was collected and frozen using the same protocol, with variations in the cryoprotectant (glycerol 5\% or 5\% DMF) and the addition or absence of cholesterol as membrane modifier $(1.5 \mathrm{mg}$ cholesterol loaded cyclodextrins for each $120 \times 106$ cells). The cryosurvival was evaluated by estimating motility using the SCA software. The vitality and membrane integrity was estimated using eosin nigrosine staining and by hypo-osmotic test respectively. Results. Sperm incubated with cholesterol had a significant effect
\end{abstract}


in improving the percentage of total motility, progressive motility, sperm velocity and percentage of rapid sperm along with a decreased percentage of sperm with slow motility. Additionally, there was an improvement in the percentage of live spermatozoa and spermatozoa with membrane integrity $(p<0.05)$. Dimethyl formamide $(D M F)$ as a cryoprotectant, improved all the evaluated parameters in comparison with glycerol $(p<0.05)$. Conclusions. Both procedures improved the evaluated parameters due to the additive effects of the cryoprotectant and the membrane modifier, but there was no interaction between these two factors.

Key words: Cholesterol, cryopreservation, dimethylfomamide (Source:CAB).

\section{INTRODUCCIÓN}

El uso de semen criopreservado en equinos ha adquirido gran importancia en las últimas décadas, debido a los beneficios que ofrece su utilización. Entre éstos se destacan la posibilidad de almacenar el material genético de reproductores sobresalientes, distribuirlo internacionalmente, controlar enfermedades venéreas y aumentar la eficiencia del sistema de cría al posibilitar la inseminación de múltiples yeguas con un solo eyaculado (1). Sin embargo, la tasa de preñez disminuye cuando se insemina con semen congelado en comparación con la utilización de semen fresco o refrigerado $(2,3)$. Esto sucede porque el proceso de congelación y descongelación es potencialmente dañino, e incluso letal, para los espermatozoides (4-7). Los daños en la estructura de la membrana plasmática y el estrés osmótico al que son sometidos los espermatozoides durante la congelación, son dos de las principales causas que disminuyen la calidad del semen después de la congelación (2, 8-10). Por esto es importante modificar las técnicas existentes enfocando los cambios hacia las etapas más críticas para mejorar los parámetros espermáticos posdescongelación con el fin de aumentar la tasa de fertilidad al utilizar semen congelado.

En el proceso de congelación ocurren daños significativos en la membrana plasmática de los espermatozoides, durante el descenso de temperatura, ésta comienza un estado de transición desde estado líquido a temperatura ambiente a un estado en gel, en el cual la membrana se vuelve más rígida y porosa (2), al haber una reorganización de los lípidos y proteínas, induciendo disrupciones y haciéndola más susceptible a moléculas extracelulares y daños producidos por cristales de hielo $(10,11)$. La relación colesterol:fosfolípidos de membrana, es una característica asociada a la sensibilidad de las células durante el descenso de la temperatura; altas cantidades de colesterol en la membrana reducen la temperatura a la que ocurre la transición de fase, manteniendo así un estado más fluido a bajas temperaturas y reduciendo potencialmente los daños $(6,12)$. El colesterol ha sido adicionado a las membranas espermáticas de varias especies, mediante el uso de ciclodextrinas (13-17). Las ciclodextrinas son oligosacaridos cíclicos con un centro hidrofóbico capaz de transportar colesterol por gradiente de concentración desde y hacia las membranas plasmáticas (13). Cuando las ciclodextrinas cargadas con colesterol (CLC) fueron adicionadas al semen bovino antes de criopreservarlo, fueron recuperados mayores porcentajes de espermatozoides móviles y con membranas intactas después de descongelar, comparados con el tratamiento control $(14,15)$. Este procedimiento también ha sido probado en semen de caballos, encontrando qué los espermatozoides tratados con colesterol mediante esta técnica aumentaron significativamente el porcentaje de movilidad, y de membranas intactas después de la criopreservación (13).

El uso de crioprotectores alternativos se ha planteado como una medida para disminuir el estrés osmótico durante el proceso de congelación de semen equino, ya que se ha reportado que el glicerol parece tener efectos tóxicos en espermatozoides de esta especie $(18,19)$. Estos efectos pueden ser disminuidos con el uso de otros compuestos con menor peso molecular como algunas amidas $(18,20)$. La dimetilformamida (DMF) ha mostrado tener resultados crioprotectores similares $(5,18)$ o superiores (21-23) al glicerol, adicionalmente, ha mostrado ser un crioprotector benéfico para espermatozoides de caballos con baja tasa de supervivencia posdescongelación, como los caballos de raza Mangalarga marchador (21). Es importante tener en cuenta que el componente genético parece influenciar las características seminales y la supervivencia posdescongelación (24). Esta investigación se llevó a cabo en caballos criollos colombianos, aprovechando su alto potencial como caballos de silla y la ausencia de datos publicados en este aspecto en este tipo de caballos. 
El objetivo de esta investigación fue evaluar el efecto del colesterol y la DMF sobre la criosupervivencia de espermatozoides de caballos criollos colombianos.

\section{MATERIALES Y MÉTODOS}

Recolección del semen. Se emplearon diez caballos criollos colombiano de los cuales a cada uno se le recolectó un eyaculado. Los criterios de inclusión dentro de la población para muestreo fueron: buena condición corporal, rango de edad entre 4 y 16 años y buen estado de salud. Las muestras de semen fueron colectadas usando la metodología de colección con vagina artificial (modelo Hannover, Minitub; Landshut, Alemania) con la ayuda de una yegua en estro. La fracción de gel fue removida con un filtro que se incorporó a la vagina artificial.

Todos los reactivos químicos fueron procedentes de Sigma-Aldrich (St. Louis, MO), excepto la metil- $\beta$-ciclodextrina de TCI América $y$, el colesterol de Alfa Aesar.

Preparación de ciclodextrina cargada con colecterol (CLC). La solución de ciclodextrina cargada con colesterol fue preparada como lo describen Purdy y Graham (14). En un tubo de ensayo se disolvieron $200 \mathrm{mg}$ de colesterol en $1 \mathrm{ml}$ de cloroformo. En otro tubo de ensayo se diluyó $1 \mathrm{~g}$ de metil- $\beta$-ciclodextrina en $2 \mathrm{ml}$ de metanol. Se adicionaron una alícuota de 450 $\mu \mathrm{l}$ de la solución de colesterol a la solución de ciclodextrina y se mezcló hasta que la solución estuviera homogénea. En una caja de Petri, se removió el solvente usando una corriente de nitrógeno gaseoso. Los cristales resultantes se dejaron secar $24 \mathrm{~h}$ y luego se pasaron a un recipiente de vidrio y permanecieron almacenados a $22^{\circ} \mathrm{C}$ hasta su uso. La solución de trabajo CLC se preparó agregando $50 \mathrm{mg}$ de CLC a $1 \mathrm{ml}$ de TALP (medio Tyrodes-AlbuminaLactato-Piruvato modificado) mezclándolo vigorosamente en un vortex, e incubándolo en un baño serológico a $37^{\circ} \mathrm{C}$ hasta el momento de ser utilizado.

Tratamientos, procesamiento, congelación y descongelación del semen. Se evaluó la concentración a cada eyaculado libre de gel (spermacue, Minitub; Landshut, Alemania) para diluirlo en TALP a una concentración final de $120 \times 10^{6}$ espermatozoides $/ \mathrm{ml}$. Posteriormente el eyaculado se dividió en cuatro alícuotas numeradas al azar según el tratamiento a realizar. El tratamiento 1 no tuvo adición de CLC y su diluyente contenía glicerol, el tratamiento 2 tuvo adición de CLC y su diluyente contenía glicerol. El tratamiento 3 no tuvo adición de CLC y su diluyente contenía DMF, el tratamiento 4 tuvo adición de CLC y su diluyente contenía DMF. Los tratamientos con colesterol, se les adicionó solución de trabajo de CLC $(1.5 \mathrm{mg}$ de CLC $/ 120 \times 10^{6}$ células) y fueron incubados a temperatura ambiente $\left(\sim 22^{\circ} \mathrm{C}\right)$ por 15 minutos. Todos los tratamientos se diluyeron a una concentración $1: 1$ (v:v) usando diluyente Kenney modificado (45\% sucrosa, $29 \%$ glucosa y $26 \%$ leche descremada), las muestras fueron centrifugadas a 400 gravedades por 12 minutos.

El sobrenadante fue removido y se estimó la concentración del precipitado, las células fueron resuspendidas en diluyente de congelación ( $60 \%$ solución lactosa al $11 \%$, $24 \%$ yema de huevo, $9 \%$ glucosa, $0.8 \%$ citrato de sodio, $0.8 \%$ EDTA, $0.2 \%$ bicarbonato de sodio) y $6 \%$ del crioprotector (tratamientos 1 y 2 glicerol y tratamientos 3 y 4 DMF) a una concentración final de $200 \times 10^{6}$ espermatozoides $/ \mathrm{ml}$. El semen se empacó en pajillas de $0.5 \mathrm{ml}$.

Posteriormente estas fueron puestas en forma horizontal sobre una gradilla de congelación de semen en una caja de porex, durante 15 minutos a $6 \mathrm{~cm}$ por encima del nivel de nitrógeno líquido, como lo describen Medeiros et al (20). Después de este tiempo, las pajillas se introdujeron en nitrógeno líquido donde permanecieron almacenadas por tres meses antes de ser descongeladas. La descongelación se hizo en agua a $37^{\circ} \mathrm{C}$ durante 30 segundos (18).

Evaluación del semen. La concentración espermática fue estimada por espectrofotometría (spermacue, Minitub; Landshut, Alemania) en una alícuota de semen fresco, el resultado de esta medición se utilizó para determinar la cantidad de diluyente y tratamiento.

Los parámetros de movilidad de espermatozoides descongelados se reportaron después de evaluar la muestra usando el software analizador de imagen SCA (Sperm Class Analyzer. Microptic SL, SCA VET 01), con la ayuda de un microscopio de contraste de fase (Nikon Eclipse 50i, Japón). Para su evaluación se diluyó el contenido de una pajilla en diluyente Kenney modificado a una relación $1: 4(\mathrm{v}: \mathrm{v})$ y se estabilizó por 10 minutos a temperatura ambiente (13). Luego se depositaron $10 \mu \mathrm{l}$ del semen diluido en una cámara Makler (Sefi Medical Instruments Ltd, Israel) y se enfocó en el microscopio de contraste de fase a un aumento de $10 \mathrm{X}$ donde fue analizado por el SCA. Al menos 500 espermatozoides fueron evaluados para cada muestra con la programación estándar (30 marcos adquiridos). 
Las variables de movilidad que se analizaron fueron: Porcentaje de movilidad, progresividad, velocidad curvilínea (VCL), velocidad lineal (VSL). El equipo consideró como espermatozoides con movilidad progresiva, aquellos que tenían una velocidad mayor a $90 \mu \mathrm{m} / \mathrm{s}$.

La integridad de membrana fue determinada con un test hipoosmótico en agua destilada, así: se incubó una alícuota de $100 \mu \mathrm{l}$ de semen en $200 \mu$ de agua destilada para una osmolaridad aproximada de $100 \mathrm{mOsm} / \mathrm{L}$, a $37^{\circ} \mathrm{C}$ durante 5 minutos (25). Se observaron 200 células por muestra bajo un microscopio de contraste de fase con un objetivo de 40X. El porcentaje de espermatozoides vivos se determinó observando extendidos seminales teñidos con colorante eosina-nigrosina modificado (citrato de sodio al 3\%), se contaron alrededor de 200 espermatozoides por muestra. Se consideraron como muertos los espermatozoides con coloración rosada y como vivos los transparentes.

Análisis estadístico. Se utilizó un diseño de bloques completos al azar con 10 repeticiones bloqueando por caballos, con la intención de controlar la posible variabilidad entre ejemplares $(6,24)$, de manera que las medidas de los tratamientos fueran comparables. Los tratamientos se organizaron con base en una estructura factorial $2 \times 2$, siendo los factores el crioprotector (niveles: glicerol o DMF) y el modificador de membrana (niveles: presencia o ausencia de colesterol). Uno de los propósitos de este diseño fue identificar sí existía interacción entre ambos factores. Se realizaron análisis de varianza para determinar la diferencia entre cada nivel de los factores en cada una de las variables respuesta de porcentajes de movilidad, porcentajes de integridad de membrana y porcentajes de vitalidad.

Al ser las variables respuesta proporciones binomiales, se requirió emplear una transformación angular en algunas de las variables para que éstas satisficieran los supuestos de normalidad. Para los análisis se utilizó el procedimiento GLM del programa estadístico SAS (versión 9.1 para Windows, 2003).

\section{RESULTADOS}

El análisis de varianza no mostró interacción entre los factores. Para las seis variables evaluadas, con 9 grados de libertad, el valor $p$ mínimo fue de 0.35 y el máximo 0.64. Por lo tanto los resultados se presentaran de manera independiente, es decir, se evaluaron los efectos simples de cada factor.

El semen tratado con colesterol como modificador de la membrana plasmática aumentó significativamente el porcentaje de espermatozoides con movilidad total y progresiva post-descongelación al ser comparado con semen no tratados. Al utilizar DMF como agente crioprotector aumentó significativamente el porcentaje de espermatozoides con movilidad total y progresiva post-descongelación al ser comparado con semen congelado con glicerol (Figura 1).
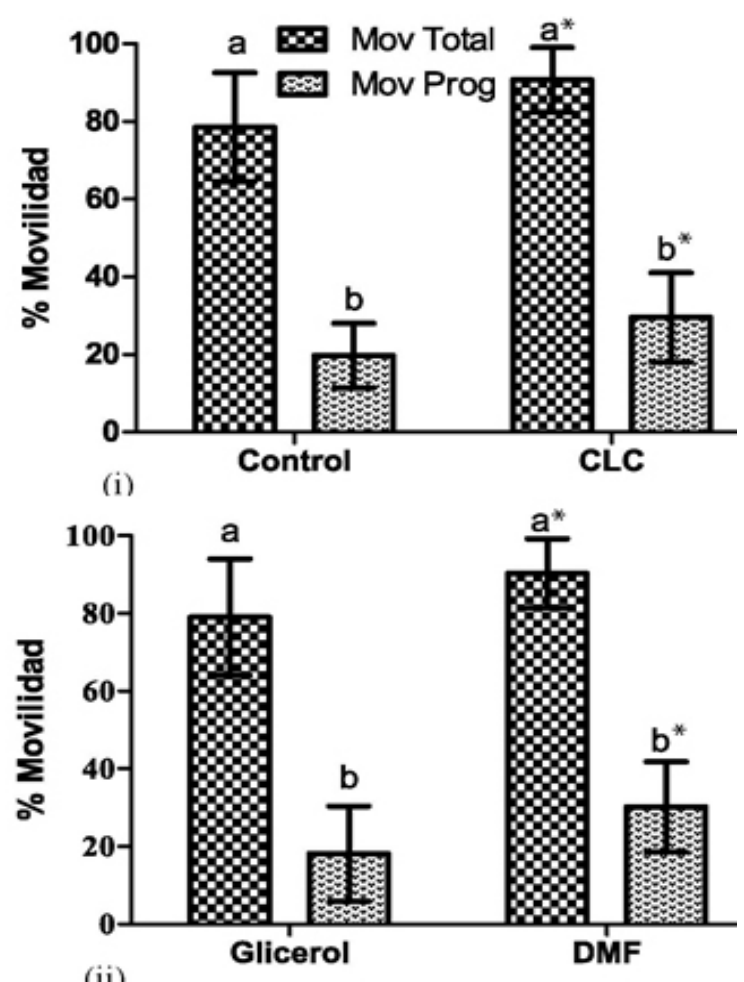

Figura1. Efecto del uso de colesterol (i) y glicerol o DMF (ii) sobre la movilidad espermática posdescongelación. Las barras de error ilustran la desviación estándar. Los asteriscos indica diferencia significativa (Note que la comparación estadística es entre barras con la misma letra) $(p<0.05)$.

Al calcular la trayectoria de cada una de las células para determinar la velocidad curvilínea y la velocidad lineal, se encontró que todos los tipos de velocidades posdescongelación fueron mayores al utilizar colesterol previo a la congelación y DMF como agente crioprotector (Figura 2).

Al adicionar colesterol y usando DMF como agente crioprotector, hubo un aumento significativo en el porcentaje de espermatozoides vivos y con membrana integra (Figura 3). 

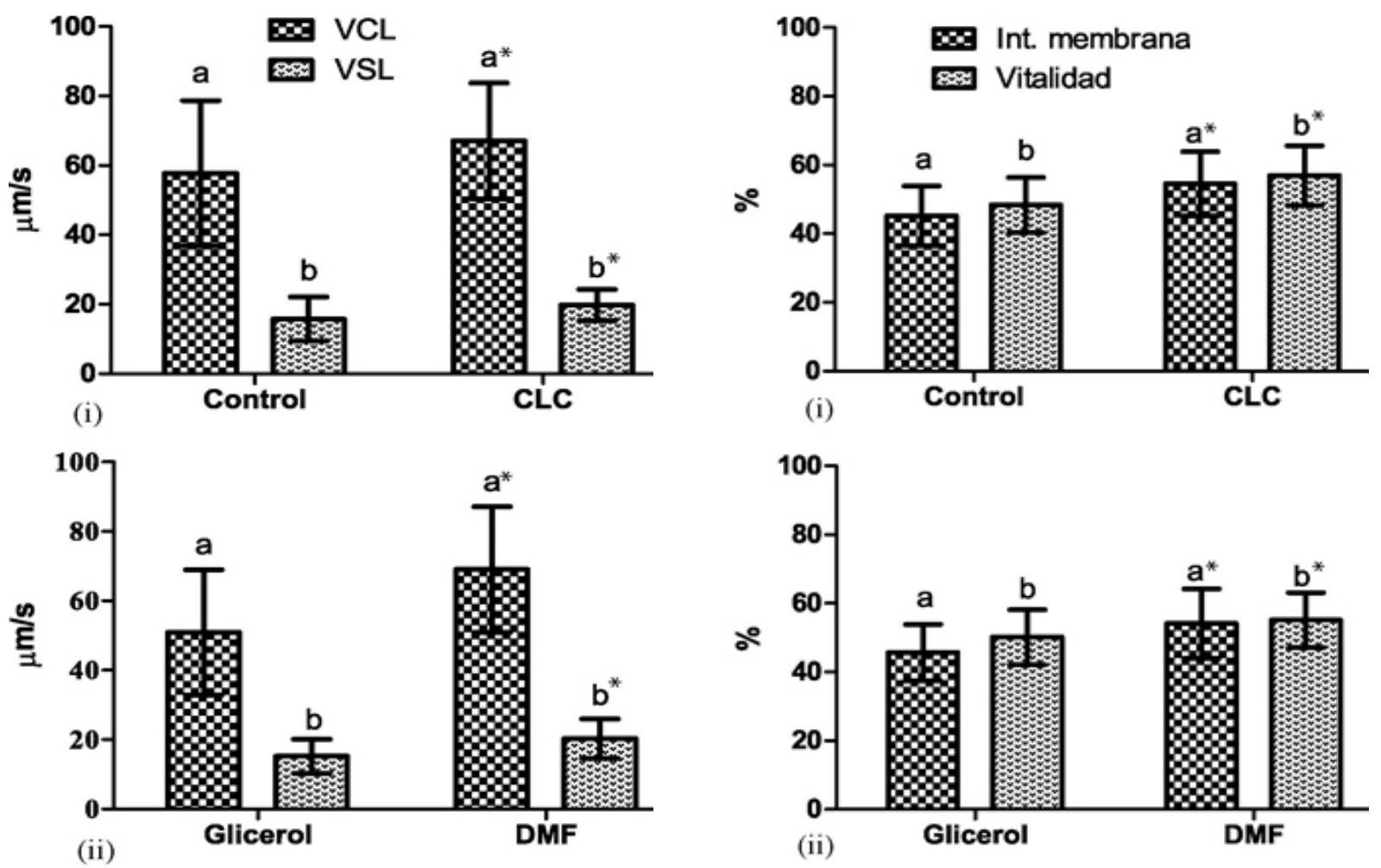

Figura2. Efecto del uso de colesterol (i) y glicerol o DMF (ii) sobre la velocidad curvilínea (VCL) y la velocidad lineal (VSL) posdescongelación. Las barras de error ilustran la desviación estándar. Los asteriscos indica diferencia significativa (Note que la comparación estadística es entre barras con la misma letra) $(p<0.05)$.

\section{DISCUSIóN}

La relación colesterol:fosfolípidos en la membrana plasmática, juega un papel fundamental en la resistencia de las células al shock térmico $(2,12)$. Las ciclodextrinas parecen proporcionar el vehículo necesario para modificar esta relación $(13-16,25)$. Cuando se incubó el semen de caballos criollos colombianos con CLC antes de la congelación, se encontró un aumento significativo en el porcentaje de: movilidad total, progresiva, espermatozoides vivos, con integridad de membrana y aumentaron las velocidades promedio posdescongelación. Estos hallazgos coinciden con el concepto de que especies que tienen alta relación molar de colesterol:fosfolípidos son más resistentes al shock térmico (26). Ha sido demostrado que al aumentar la concentración de colesterol en la membrana se disminuye la temperatura de transición y de esta manera la membrana permanece más fluida (6) siendo menos susceptible a daños mecánicos, lo cual se refleja notablemente en su integridad, en la que los presentes resultados coinciden con los

Figura3. Efecto del uso de colesterol (i) y glicerol o DMF (ii) sobre el porcentaje de vitalidad e integridad de membrana posdescongelación, barras de error ilustran la desviación estándar. Los asteriscos indica diferencia significativa (Note que la comparación estadística es entre barras con la misma letra) $(p<0.05)$.

de otras investigaciónes similares en otras razas equinas $(13,25,27)$.

Para los parámetros de movilidad espermática posdescongelación el efecto del colesterol es controversial. Los presentes resultados indican un aumento en la movilidad, coincidiendo con varias investigaciones semejantes en caballos $(13,28)$ y en toros $(14,16)$, sin embargo otros trabajos en caballos no encontraron tal diferencia $(25,27)$; esto podría ser debido a que la reducción de la movilidad posdescongelación no necesariamente se debe atribuir a daños en la membrana (2).

Zahn et al (27) encontraron una disminución en la fertilidad posdescongelación de espermatozoides tratados con CLC, pues al alterarse la composición de la membrana, además de afectarse la respuesta de los espermatozoides al proceso de congelación, también se afecta su capacitación y posterior reacción acrósomica, al ser el flujo del colesterol una de las primeras etapas de la capacitación espermática (29). Spizziri et al (30), no encontraron diferencias en fertilidad al 
inseminar yeguas con semen tratado y no tratado con CLC. Se deben llevar a cabo posteriores pruebas de fertilidad para determinar el momento óptimo de inseminación con semen tratado con colesterol precongelación, porque al aumentar la cantidad de colesterol en la membrana, el tiempo de capacitación aumenta y por ende el tiempo de inseminación debe adelantarse. Una alternativa planteada recientemente es la adición de ciclodextrinas posdescongelación para que por gradiente de concentración vuelvan a extraer el colesterol de las membranas (25). Sin embargo, en ese caso no se obtuvieron los resultados esperados.

El glicerol es el agente crioprotector más frecuentemente utilizado para la congelación de semen en varias especies incluyendo caballos (10). A pesar de esto en el presente experimento todos los parámetros seminales evaluados mejoraron al utilizar la DMF como crioprotector alterno al glicerol. La capacidad superior como agente crioprotector de la DMF es consistente con trabajos previos $(18,20,21,23,31)$. Para que un crioprotector sea efectivo debe tener bajo peso molecular, ser soluble en agua y causar baja toxicidad (32). El glicerol tiene mayor peso molecular que la DMF (92 vs 73), esto le permite a la DMF penetrar la membrana más rápido produciendo así un cambio en el volumen celular menos drástico o de menor magnitud que el que puede producir el glicerol, lo cual se pudo haber visto reflejado en el mayor porcentaje de movilidad, espermatozoides vivos e íntegros encontrados cuando se utilizó DMF.

Además del estrés osmótico que ejerce el glicerol en los espermatozoides, se ha sugerido que también causa toxicidad por medio de efectos directos sobre la membrana y el metabolismo celular (10). Se ha descrito que el glicerol altera la bicapa lipídica modificando la tasa de permeabilidad de agua y los movimientos de iones transmembranales, igualmente el glicerol interacciona con proteínas y lipoproteínas de membrana y aumenta la demanda energética celular (10). En la revisión realizada no se ha reportado toxicidad de la dimetilformamida como agente crioprotector de espermatozoides.

Los presentes hallazgos, en cuanto a la acción superior de la DMF coinciden con los de Gómes et al (21) y Gibb et al (33) quienes compararon la acción de amidas y glicerol como agentes crioprotectores en la congelación de semen en caballos, encontrando que los espermatozoides congelados con DMF tuvieron una movilidad total superior a los congelados con glicerol y al de otros tratamientos, sin embargo Gibb et al (33), solo encontraron diferencia en movilidad y no en integridad de membrana. Los experimentos que evalúan el efecto del glicerol contra DMF y aplicados conjuntamente tuvieron resultados controversiales. Vidament et al (23) encontraron que la movilidad no mejoraba al utilizar la combinación de estos crioprotectores y que el uso de la DMF presentaba resultados comparables con los del glicerol, mientras que Medeiros et al (20) reportan que la asociación de glicerol con DMF presentó resultados de movilidad mejores que los del glicerol solo, pero esta asociación no tuvo diferencia con el tratamiento que utilizó únicamente DMF.

El uso de DMF como agente crioprotector es una alternativa para mejorar la movilidad espermática, la integridad de membrana y la vitalidad, especialmente si se tiene en cuenta que se ha reportado que el glicerol tiene un efecto contraconceptivo en yeguas (21). Son necesarias pruebas posteriores de fertilidad para verificar si el efecto benéfico sobre las características espermáticas posdescongelación se manifiestan en una mayor tasa de concepción.

El análisis estadístico indicó que no hubo interacción entre la adición de colesterol y los dos crioprotectores evaluados. Se ha reportado que el colesterol además de prevenir los daños mecánicos disminuye el estrés osmótico $(9,25)$ debido a que al aumentar la fluidez de las membranas a bajas temperaturas, aumenta también la permeabilidad de los crioprotectores a través de ella $(9,34)$; al parecer en este caso la diferencia en el peso molecular de los crioprotectores no fue lo suficientemente grande para influenciar los parámetros posdescongelación. El tratamiento de espermatozoides al que le se le adicionó colesterol y DMF como agente crioprotector tuvo el mejor rendimiento de los parámetros evaluados, debido únicamente a los efectos aditivos de ambos.

\section{Agradecimientos}

A la empresa Embryotransfer LTDA y al laboratorio del procesamiento de semen, San Pablo de la Universidad Nacional por el financiamiento de este trabajo. Al Centro de Investigación Piscícola de la Universidad de Córdoba CINPIC por permitirnos la evaluación de las muestras en su laboratorio. 


\section{REFERENCIAS}

1. Loomis PR. The equine frozen semen industry. Anim Reprod Sci 2001; 68:(34):191-200.

2. Watson PF. The causes of reduced fertility with cryopreserved semen. Anim Reprod Sci 2000;60-61:481-92.

3. Vidament $M$, Dupere $A M$, Julienne $P$, Evain A, Noue P, Palmer E. Equine frozen semen: freezability and fertility field results. Theriogenology 1997; 48(6):907-17.

4. Crockett EC, Graham JK, Bruemmer JE, Squires EL. Effect of cooling of equine spermatozoa before freezing on postthaw motility: preliminary results. Theriogenology 200; 55(3):793-803.

5. Henry M, SnoecK PPN, Cottorello ACP. Post-thaw spermatozoa plasma membrane integrity and motility of stallion semen frozen with different cryoprotectants. Theriogenology 2002; 58:245-48.

6. Amann RP, Pickett BW. Principles of cryopreservation and a review of cryopreservation of stallion spermatozoa. Equine Vet Sci 1987; 7:28.

7. O'Connell M, McClure N, Lewis SE. The effects of cryopreservation on sperm morphology, motility and mitochondrial function. Hum Reprod 2002;17(3):704-9.

8. Meyers SA. Spermatozoal response to osmotic stress. Anim Reprod Sci 2005; 89(1-4):57-64.

9. Glazar AI, Mullen SF, Liu J, Benson JD, Critser JK, Squires EL, et al. Osmotic tolerance limits and membrane permeability characteristics of stallion spermatozoa treated with cholesterol. Cryobiology 2009; 59(2):201-6.

10. Hammerstedt RH, Graham JK, Nolan JP. Cryopreservation of mammalian sperm: what we ask them to survive. J Androl $1990 ; 11(1): 73-88$.

11. Mazur P. The role of intracellular freezing in the death of cells cooled at supraoptimal rates. Cryobiology 1977; 14(3):251-72.
12. Darin-Bennett $A$, White IG. Influence of the cholesterol content of mammalian spermatozoa on susceptibility to cold-shock. Cryobiology 1977; 14(4):466-70.

13. Moore AI, Squires EL, Graham JK. Adding cholesterol to the stallion sperm plasma membrane improves cryosurvival. Cryobiology 2005; 51(3):241-9.

14. Purdy PH, Graham JK. Effect of cholesterolloaded cyclodextrin on the cryosurvival of bull sperm. Cryobiology 2004; 48(1):36-45.

15. Amorim EA, Graham JK, Spizziri B, Meyers $M$, Torres CA. Effect of cholesterol or cholesteryl conjugates on the cryosurvival of bull sperm. Cryobiology 2009; 58(2):210-4.

16. Purdy $\mathrm{PH}$, Graham JK. Effect of adding cholesterol to bull sperm membranes on sperm capacitation, the acrosome reaction, and fertility. Biol Reprod 2004; 71(2):522-7.

17. Moraes EA, Graham JK, Torres CA, Meyers M, Spizziri B. Delivering cholesterol or cholestanol to bull sperm membranes improves cryosurvival. Anim Reprod Sci $2010 ; 118(2-4): 148-54$.

18. Squires EL, Keith SL, Graham JK. Evaluation of alternative cryoprotectants for preserving stallion spermatozoa. Theriogenology 2004; 62(6):1056-65.

19. Demick DS, Voss JL, Pickett BW. Effect of cooling, storage, glycerolization and spermatozoal numbers on equine fertility. J Anim Sci 1976; 43(3):633-7.

20. Medeiros AS, Gomes GM, Carmo MT, Papa FO, Alvarenga MA. Cryopreservation of stallion sperm using different amides. Theriogenology 2002; 58:4.

21. Gomes GM, Jacob JCF, Medeiros AS, Papa FO, Alvarenga MA. Improvement of stallion spermatozoa preservation with alternative cryoprotectants for the Mangalarga Marchador breed Theriogenology 2002; 58:2. 
22. Alvarenga MA, Keith SL, Landim-Alvarenga FC, Squires EL, editors. Alternative cryoprotectors for freezing stallion spermatozoa. 14th International Congress on Animal Reproduction; 2002.

23. Vidament $M$, Daire $C$, Yvon JM, Doligez $P$, Bruneau B, Magistrini M, et al. Motility and fertility of stallion semen frozen with glycerol and/or dimethyl formamide. Theriogenology 2002; 58:249-51.

24. Dowsett KF, Knott LM. The influence of age and breed on stallion semen. Theriogenology 1996; 46(3):397-412.

25. Oliveira $\mathrm{CH}$, Vasconcelos $A B$, Souza $F A$, Martins-Filho OA, Silva MX, Varago FC, et al. Cholesterol addition protects membrane intactness during cryopreservation of stallion sperm. Anim Reprod Sci 2010; 118(2-4):194-200.

26. Parks JE, Lynch DV. Lipid composition and thermotropic phase behavior of boar, bull, stallion, and rooster sperm membranes. Cryobiology 1992; 29(2):255-66.

27. Zahn FS, Papa FO, Jr. DA. Cholesterol incorporation on equine sperm membrane: effects on post-thaw sperm parameters and fertility. Theriogenology 2002; 58:237-40.

28. Combes GB, Varner DD, Schroeder F. Effect of cholesterol on the motility and plasma membrane integrity of frozen equine spermatozoa after thawing. J Reprod Fertil Suppl 2000; 56:127-32.
29. Neild DM, Gadella BM, Chaves MG, Miragaya $\mathrm{MH}$, Colenbrander B, Aguero A. Membrane changes during different stages of a freeze-thaw protocol for equine semen cryopreservation. Theriogenology 2003; 59(8): 1693-705.

30. Spizziri BE, Fox MH, Bruemmer JE, Squires EL, Graham JK. Cholesterol-loadedcyclodextrins and fertility potential of stallions spermatozoa. Anim Reprod Sci 2010; 118(2-4):255-64.

31. Alvarenga MA, Papa FO, Landim-Alvarenga FC, Medeiros AS. Amides as cryoprotectants for freezing stallion semen: a review. Anim Reprod Sci 2005; 89(1-4):105-13.

32. Pegg DE. Principles of cryopreservation. In: Day JG, Stacey GN, editors. Cryopreservation and Freeze-Drying Protocols. 2 ed. Totowa, New Jersey: Humana Press; 2007.

33. Gibba Z, Morrisb LHA, Maxwella WMC, C.G. G. Dimethyl formamide improves the postthaw characteristics of sex-sorted and nonsorted stallion spermatozoa. Anim Reprod Sci 2010; 121(1-2):216-7.

34. Moce E, Blanch E, Tomas C, Graham JK. Use of cholesterol in sperm cryopreservation: present moment and perspectives to future. Reprod Domest Anim 2010; 45(Suppl 2):57-66. 